\title{
Crocin and voluntary exercise promote heart angiogenesis through Akt and ERK1/2 signalling in type 2 diabetic rats
}

\author{
Dariushnejad $\mathrm{H}^{1}$, Mohammadi $\mathrm{M}^{2}$, Ghorbanzadeh $\mathrm{V}^{3}$ \\ Razi Herbal Medicines Research Center, Lorestan University of Medical Sciences, Khorramabad, Iran. \\ vghorbanzadeh@gmail.com
}

\section{ABSTRACT}

OBJECTIVES: The aim of this study was to evaluate the effects of crocin and voluntary exercise on heart angiogenesis through Akt and ERK1/2 signalling.

METHODS: Animals were divided into 4 groups as follows: diabetes (Dia), diabetic-crocin (Dia-Cro), diabeticvoluntary exercise (Dia-Exe), diabetic-crocin-voluntary exercise (Dia-Cro-Exe). Type 2 diabetes was induced by high-fat diet (4 weeks) and injection of streptozotocin (STZ) (i.p., $35 \mathrm{mg} / \mathrm{kg}$ ). Animals received crocin orally $(50 \mathrm{mg} / \mathrm{kg}$ ), voluntary exercise was performed alone or together with crocin for 8 weeks. Akt and ERK1/2 levels were measured by ELISA and CD31 was detected by immunohistochemistry.

RESULTS: Akt and ERK1/2 levels in crocin and exercise groups were significantly higher than in diabetic group. Levels of Akt and ERK1/2 proteins and CD31 immunostaining were also significantly higher in crocin-voluntary exercise group in comparison to the other groups.

CONCLUSION: Our results revealed that treatments with crocin and voluntary exercise had synergistic effect on angiogenesis. Protective effects of these interventions are probably through the activation of Akt and ERK $1 / 2$ signalling pathways in the heart of diabetic rats (Fig. 3, Ref. 40). Text in PDF www.elis.sk. KEY WORDS: type 2 diabetes, crocin, voluntary exercise, Akt; ERK1/2.

\section{Introduction}

Type 2 diabetes mellitus is a growing public health problem affecting millions of people worldwide. Its symptoms include progressive hyperglycemia, insulin resistance, and reduction in insulin secretion by pancreatic $\beta$-cells in response to increased blood glucose $(1,2)$. Patients with diabetes have a 4-fold greater risk of developing coronary arterial disease (3). Diabetes causes endothelial dysfunction and thereby contributes to the development of coronary artery disease (4). The molecular mechanisms underlying the impairment of angiogenesis in type 2 diabetes have remained largely unidentified. Recent studies revealed that diabetic abnormal angiogenesis was closely associated with an impaired Akt and ERK1/2 signalling (5). Hyperglycemia alters Akt and ERK1/2 signalling and leads to endothelial cell proliferative dysfunction (6). In diabetic patients, the expression of the vascular endothelial growth factor (VEGF) receptors decreases through reduced Akt phosphorylation, which in the long term worsens ischemic state. VEGF plays an important role in angiogenesis that activates cell

${ }^{1}$ Department of Medical biotechnology, Lorestan University of Medical Sciences, Khorramabad, Iran, ${ }^{2}$ Drug Applied Research Center of Tabriz University of Medical Sciences, Tabriz, Iran, and ${ }^{3}$ Razi Herbal Medicines Research Center, Lorestan University of Medical Sciences, Khorramabad, Iran

Address for correspondence: V. Ghorbanzadeh, Dr, Razi Herbal Medicines Research Center, Lorestan University of Medical Sciences, Khorramabad, Iran.

Phone: +986633204005 proliferation via ERK1/2 $(7,8)$. On the other hand, expression of ERK1/2 protein, an activator of VEGF induced cell proliferation, was decreased in diabetic animals. Type 2 diabetes is associated with reduced Akt and ERK1/2 signalling along with impaired angiogenesis and collateral blood vessel formation.

Crocin, an active component of Crocus sativus (saffron), has been an important topic for research recently. Crocin exhibits a variety of effects including a decrease in the fasting blood glucose, HbAlc, lipid profile, and it also increases serum insulin levels (9). Furthermore, crocin has a protective effect on hypoxic damage of myocardial cells by elevation of VEGF expression (10). Active component of saffron enhances angiogenesis by a response of endodermic cells to VEFG (11). However, angiogenesis signalling pathways have not been extensively studied for understanding crocin- induced cardioprotection, particularly in diabetic animal models.

Exercise is an applicable treatment modality for diabetic patients. Training could also improve coronary vascular function by suppressing oxidative stress and inflammation in a type 2 diabetes (12). In response to training, two cellular pathways have been implicated in cellular growth: ERK and Akt (13). Aerobic exercise improves aging-induced downregulation of VEGF angiogenic signalling via phosphorylation of Akt protein in the heart, thereby contributing to the training-induced angiogenesis (14). Exercise reduced the heart disease risk and improved diabetic-mediated cardiovascular complications (15). Although many beneficial effects were reported for exercise, exhaustive exercise induces oxidative stress through a production of reactive oxygen species and can 


\section{$757-761$}

cause damage to organs (16). Voluntary exercise is ranged in mild /moderate exercise and is a useful therapeutic approach in cardiovascular diseases. In the animal model of voluntary exercise, the animal has free entrance to a running wheel and utilizes the wheel according its physiological threshold for physical activity (17).

Based on these observations, this experiment was designed to test the hypothesis that crocin and voluntary exercise can improve the heart angiogenesis via Akt and ERK1/2 signalling in diabetic rats.

\section{Material and methods}

\section{Animals}

Male Wistar rats (200-250 g) were obtained from Tabriz medical faculty (Iran-Tabriz). They were housed four per cage except the rats in the exercise groups that were alone. Animals were housed in a room with a constant temperature of $24^{\circ} \mathrm{C}$, a relative humidity of $50 \%$, and a $12 \mathrm{~h}$ dark / light cycle with access to food and water ad libitum. This study was approved by the Animal Ethics Committee (document number 92197) in accordance with the instruction for the care and use of laboratory animals prepared by Tabriz University of Medical Sciences (Iran-Tabriz).

\section{Experimental design}

All rats were made diabetic by a high fat diet and STZ injection, then they were randomly divided into four groups $(n=7)$. Group 1 received a high-fat diet (4 weeks) and low dose of STZ $(35 \mathrm{mg} / \mathrm{kg}$ ) as a diabetic group (Dia). Group 2 received a single dose of crocin $(50 \mathrm{mg} / \mathrm{kg})$ for eight weeks after confirmation of Diabetes (Dia-Cro). Group 3 performed voluntary exercise for eight weeks after confirmation of Diabetes (Dia-Exe). Group 4 received crocin and simultaneously performed voluntary exercise for eight weeks after confirmation of Diabetes (Dia-Cro-Exe). Crocin (Sigma) was administered $(50 \mathrm{mg} / \mathrm{kg}) 6$ days a week for 8 weeks. For the assessment of voluntary exercise, rats were housed individually in a cage containing a wheel (1.00 m circumference, Tajhiz Gostar). This stainless-steel running wheel was equipped with a digital magnetic counter that was activated by wheel rotation. Each exercising rat had a separate running wheel in its cage that allowed to run voluntarily during 8 weeks of the study. The rats, whose running distance was lower than $\sim 2000 \mathrm{~m}$ per day were eliminated before statistical analysis (18).

\section{Development of type 2 diabetes}

After1 week ad libitum exposure to drinking water and standard rat pellet diet, rats were fed with high fat diet comprising 22 $\%$ fat, carbohydrate $48 \%$ and protein $20 \%$ in blend with standard laboratory chow consisting of $5 \%$ fat, $53 \%$ carbohydrate and $23 \%$ protein for 4 weeks. After the period of dietary manipulation, a single low dose of STZ $(35 \mathrm{mg} / \mathrm{kg}$, dissolved in $0.1 \mathrm{M}$ sodium citrate buffer at $\mathrm{pH} 4.4$ ) was injected into each rat intraperitoneally (Sigma) (19). After 72 hours of streptozotocin administration, fasting blood glucose level was checked by using a portable glucometer (Glucoplus Inc., Quebec, Canada) in the blood collected from the tail veins. Animals with fasting blood glucose value $>300 \mathrm{mg} / \mathrm{dl}$ were considered diabetic. Animals with fasting blood glucose concentration $<300 \mathrm{mg} / \mathrm{dl}$ were excluded from the study (20).

\section{Heart tissue preparation for Akt and ERK1/2 and CD31}

On the final day of the experiment, rats were sacrificed under anaesthesia with ketamine/xylazine (88/10 mg/kg, i.p.). Heart tissue was immediately removed and washed with saline $0.9 \%$. Hearts were excised, frozen in liquid nitrogen and stored at deep freeze $\left(-70^{\circ} \mathrm{C}\right)$ for later measurements.

\section{Akt and ERK1/2 assays}

Heart tissue samples were weighted $(0.1 \mathrm{~g})$, homogenized in PBS (pH 7.2-7.4) and centrifuged for $20 \mathrm{~min}$ at the speed of 3000 rpm and $4{ }^{\circ} \mathrm{C}$. Then supernatants were collected in a new tube and p-Akt (Cat. No. CK-E91385; Eastbiopharm Co., Ltd., Hangzhou, China), p-ERK1/2 (PT202/Y204) (Abcam, Cambridge, U.K, product code: ab176640) levels were measured using sandwich rat ELISA kits. The ELISA assay was performed according to the manufacturer's instructions and normalized to the total protein concentration for each sample, as determined by the Bradford assay.

\section{Immunohistochemical assessments}

For the investigation of angiogenesis in the heart tissue, samples from left ventricle were immersed into $10 \%$ formalin after excision, embedded in paraffin, and cut into $4 \mu \mathrm{m}$-thick slices. Sections were deparaffinized in xylene and dehydrated in a graded series of ethanol. Slides were incubated sequentially in proteinase $\mathrm{K}$ and treated by $0.3 \%$ hydrogen peroxide for blocking endogenous peroxidase activity. Sections were overlaid by primary antibody CD31 (Santa Cruz Biotechnology (Santa Cruz, CA, USA) a marker of angiogenesis and incubated at $+4{ }^{\circ} \mathrm{C}$ overnight. Sections were then washed and incubated with standard avidin-biotin complex (ABC; Santa Cruz Biotechnology) according to the manufacturer's instructions. Finally, sections were assessed by light microscope (Olympus BX 40, Japan) with an independent pathologist (21). Capillaries were visualized in the myocardium as a brown precipitate. Vascular structures positive for CD31 were counted for 5 to 6 slides per animal and 10 fields per slide.

\section{Statistical analysis}

Statistical analysis was performed using SPSS v.18.0 (IBM Corp., Armonk, NY, USA). Between group differences were analysed using two-way analysis of variance (ANOVA). Post-hoc analysis for Akt and ERK data were Tukey's test. The results are reported as the means $\pm \mathrm{SEM}$, and were considered significant at $\mathrm{p}<0.05$.

\section{Results}

\section{Effect of crocin and voluntary exercise on Akt levels}

After 8 weeks of treatment of diabetic rats with crocin and voluntary exercise, P-Akt levels in heart tissue in Dia-Cro and Dia-Exe were significantly $(\mathrm{p}<0.05, \mathrm{p}<0.01$ respectively) higher than in Dia group (Fig. 1). In the crocin combined with exercise group, the heart P-Akt levels were significantly increased compared to 


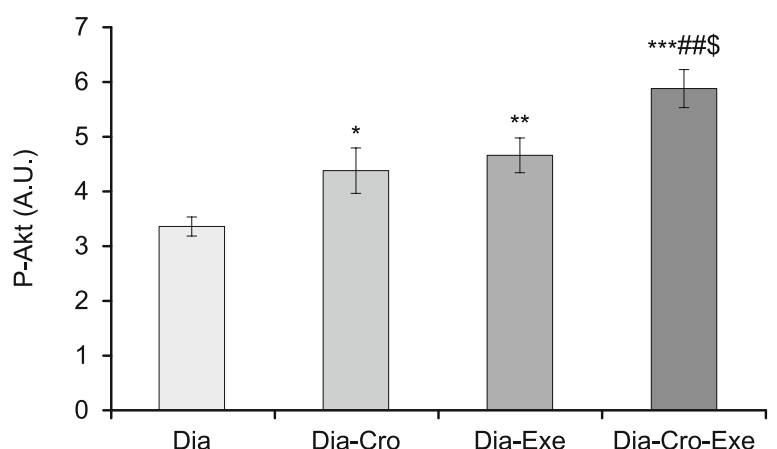

Fig. 1. Effect of crocin combined with voluntary exercise on P-Akt protein levels using ELISA method. Data are shown as mean \pm S.E.M. for $\mathrm{n}=7$ animals. ${ }^{*} \mathrm{p}<0.05,{ }^{* * *} \mathrm{p}<0.01$ and ${ }^{* * * *} \mathrm{p}<0.001$ indicates significant change compared with diabetic group. ${ }^{\#} p<0.01$ indicates significant change compared with crocin group. ${ }^{s} \mathrm{p}<0.05$ indicates significant change compared with exercise group.

diabetic animals $(\mathrm{p}<0.001)$. A significant difference was found between Dia-Cro-Exe group and Dia-Cro $(\mathrm{p}<0.01)$ or Dia-Exe groups $(\mathrm{p}<0.05)$. Figure 1 indicates that crocin combined with voluntary exercise had an additive effect in Akt protein levels in heart tissue.

\section{Effect of crocin and voluntary exercise on ERK1/2}

According to the Figure 2, two-way ANOVA indicated that treatment with crocin and exercise significantly enhanced the ERK1/2 levels in Dia-Cro ( $p<0.01)$, Dia-Exe $(\mathrm{p}<0.01)$, and Dia-Cro-Exe $(\mathrm{p}<0.001)$ in comparison with the diabetic group. Also, ERK1/2 levels in Dia-Cro-Exe group were significantly higher than those of the other treatment groups $(\mathrm{p}<0.001)$. These

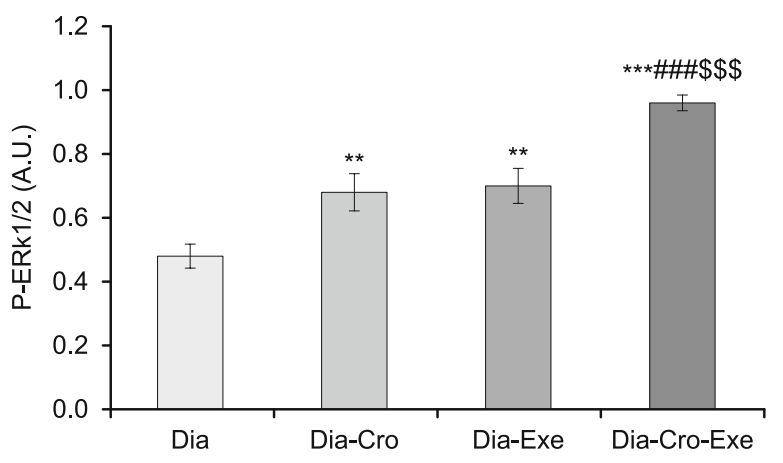

Fig. 2. Show the effect of crocin combined with voluntary exercise on P-ERK1/2 protein level using ELISA method. Data are shown as mean \pm S.E.M. for $n=7$ animals. ${ }^{* *} \mathbf{p}<0.01$ and ${ }^{* * * *} \mathbf{p}<0.001$ indicates significant change compared with diabetic group. ${ }^{\# \#} p<0.05$ indicates significant change compared with crocin group and ${ }^{\$ \$ \$} p<0.001$ indicates significant change compared with exercise group.

findings exhibit that $\mathrm{p}$-ERK1/2 increased by crocin and voluntary exercise in the diabetic groups.

\section{Effect of crocin and voluntary exercise on angiogenesis}

Immunohistochemical staining for CD31 in the cardiac sections are shown in Figure 3. CD31 was present in the diabetic group treated with crocin and voluntary exercise. There was a significant increase in CD31 immunostaining in the cardiac tissue in the crocin-exercise-treated group $(\mathrm{p}<0.001)$, when compared to the diabetic group (Fig. 3 A, D). Also, treatment of diabetic animals with crocin and voluntary exercise alone, significantly elevated angiogenesis compared to the diabetic group (all, $\mathrm{p}<$ 0.001) (Fig. 3 A, B, C).
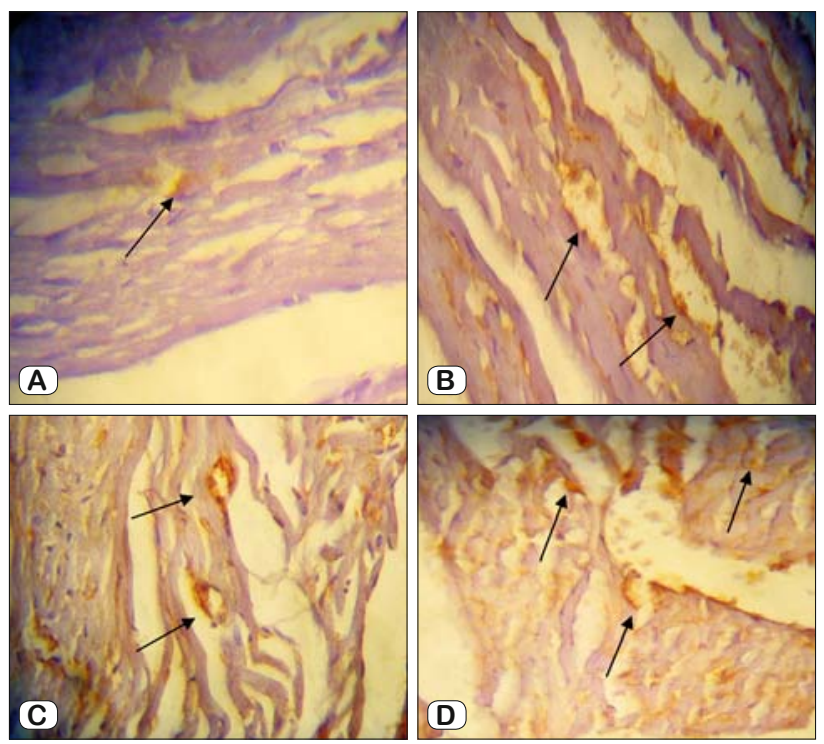

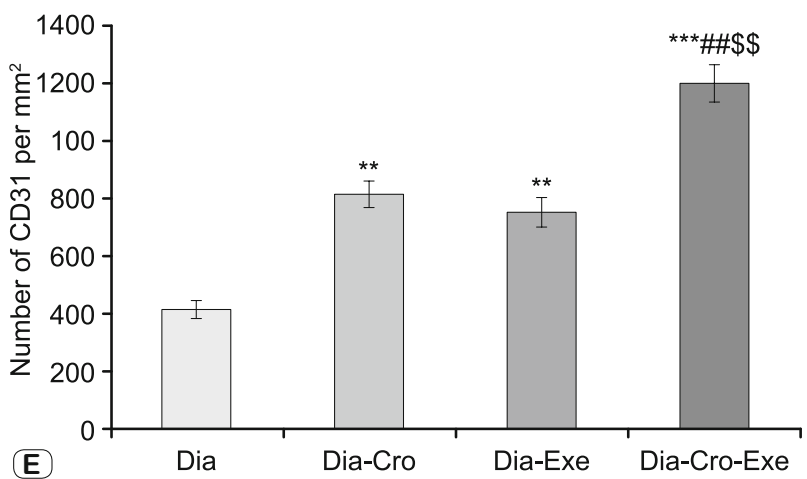

(E)
Dia-Cro
Fig. 3. Representative images of CD31 staining (brown) in coronary vessels of non-treated and treated diabetic rats. Magnification $\times 400$. A: Dia group. B: Dia-Cro group. C: Dia-Exe. D: Dia-Cro-Exe group. E: Quantification of CD31-positive cells in the diabetic groups. Data are mean \pm SEM. ${ }^{*} \mathbf{p}<0.05,{ }^{* * *} \mathbf{p}<0.001$ indicates significant change compared with diabetic group, ${ }^{\# \#} \mathbf{p}<0.01$ indicates significant change compared with crocin group and ${ }^{\mathrm{ss}} \mathrm{p}<0.01$ indicates significant change compared with exercise group. 


\section{Discussion}

The present study showed that crocin and voluntary exercise treatment increased the Akt and ERk1/2 activation in diabetic rat heart. In addition, they stimulated angiogenesis in heart tissue of diabetic rats. For the first time, our findings demonstrated that heart angiogenesis and its upstream molecules including Akt and ERK1/2 were upregulated in response to crocin combined with voluntary exercise in rats with high-fat diet-induced type 2 diabetes. Type 2 diabetes represented a formidable unmet medical health problem with an elevated risk of cardiovascular disease. In this pathologic case, there is an inadequate collateral vascular formation that ultimately results in enhanced cardiovascular morbidity and mortality rates (4). Based on previous studies it was found that in diabetes, angiogenesis is regulated dependently on organ-tissue, and cell type-specific manner (22). In the diabetic heart tissue, VEGF (pro-angiogenic molecules) signalling pathways are disturbed and collateral vessel formation is interrupted (23). VEGF exerts many of its effects via the AKT and ERK1/2 pathways (24). During developmental vasculogenesis, the AKT pathway regulates venous specification, whereas the ERK pathway regulates arterial specification (25). VEGF activates AKT via a phosphatidylinositol 3-kinase (PI 3-kinase)-dependent pathway, and this downstream effecter may play a principal role in the "outstanding" biological processes of VEGF, such as: endothelial cell proliferation, migration, and survival (26). There are several lines of evidence indicating that under diabetic condition, the expression level of phosphorylated Akt and ERK1/2 is decreased in cardiomyocytes (27), in spite of high level of VEGF (28). Our results proved that crocin combined with voluntary exercise enhanced angiogenesis and its upstream components of angiogenic signalling such as: Akt and ERK1/2. Previous studies showed that training promotes cardiac angiogenesis by enhancing the angiogenic pathways such as: ERK1/2 and PI3K/Akt both in healthy and obese animals $(29,30)$. Research confirmed that the expression levels of VEGF-A and VEGFR-2 were increased in saffron therapy group and it was suggested that the protective effects of saffron might be related to its ability to promote angiogenesis $(11,31)$. Cardioprotective effects of crocin were reported in some studies that are related to modulation of antioxidant activities and cardiac biomarkers (32). Crocin prevented retinal ischemia/reperfusion injury in retinal ganglion cells through the PI3K/Akt signalling pathway (33). Saffron strongly enhanced the phosphorylation of ERK in muscle cells and increased insulin sensitivity. Also, when the cells were exposed to saffron and insulin simultaneously, PI3K/Akt pathway was more greatly stimulated compared to those of individual treatments (34). Several rationales may be considered for this crocin- exercise-induced upregulation of Akt and ERK1/2. One possible elucidation for the ameliorated phosphorylation levels of Akt and ERK1/2 in heart tissue of the diabetic rat might be a mechanism related to glycemia (35). It was reported that hyperglycemia decreased Akt and ERK1/2 activities and further inhibited the proliferation and migration of cells necessary for neovascularization (36). Since it was found that normalized glycemia brought the phosphorylation and activity of Akt and ERK1/2 to a normal level (37), so our results that crocin combined with exercise increased P-Akt and P-ERK 1/2 levels could support this hypothesis. An additional reason for upregulation of Akt and ERK1/2 in heart tissue of diabetic rats by crocin-exercise may act via activating mitogen-activated protein kinase (MAP kinase/ ERK1/2) and PI3K/Akt $(34,38,39)$. Furthermore, there is a crosstalk between MAPK, PI3K pathways and hypoglycemic effects. Activation of these pathways promote the translocation of GLUT4 from an intracellular pool to the cell surface, which in turn can lead to an improvement of insulin stimulated glucose uptake and subsequently reduce glucose levels in diabetic cases (40).

Accordingly, training and crocin administration might be beneficial to enhance the cardiac survival in diabetic heart by ERK1/2 and Akt signalling pathways. This is the first report regarding increased angiogenesis due to administration of crocin combined with voluntary exercise through activation of Akt and ERK1/2 in heart tissue of type 2 diabetic rats. This study represents an initial step toward unravelling the mechanisms that underlie angiogenesis induced by crocin combined with voluntary exercise in type 2 diabetic model. In conclusion, our findings suggest that crocin combined with voluntary exercise can reverse reduced angiogenesis by high glucose in heart tissue of type 2 diabetes. In addition, crocin and voluntary exercise induce coronary angiogenesis by activating the Akt and ERK signalling pathways. Indeed, combination of voluntary exercise with crocin administration in type2 diabetic rats has beneficial effects compared to each treatment.

\section{References}

1. Addin EN, Fonseca VA. Defining and characterizing the progression of type 2 diabetes. Diabet Care 2009; 32 (Suppl 2): S151-S156.

2. Guthrie RA, Guthrie DW. Pathophysiology of diabetes mellitus. Crit Care Nurs Quart 2004; 27 (2): 113-125.

3. Kornowski R, Mintz GS, Kent KM, Pichard AD, Satler LF, Bucher TA, Hong MK, Popma JJ, Leon MB. Increased Restenosis in Diabetes Mellitus After Coronary Interventions Is Due to Exaggerated Intimal Hyperplasia A Serial Intravascular Ultrasound Study. Circulation 1997; 95 (6): 1366-1369.

4. Abacı A, Oğuzhan A, Kahraman S, Eryol NK, Ünal Ş, Arınç H, Ergin A. Effect of diabetes mellitus on formation of coronary collateral vessels. Circulation 1999; 99 (17): 2239-2242.

5. Huisamen B. Protein kinase B in the diabetic heart. Biochem Diabet Atheroscler: Springer; 2003. p. 31-38.

6. Varma S, Lal BK, Zheng R, Breslin JW, Saito S, Pappas PJ, Hobson RW, Durán WN. Hyperglycemia alters PI3k and Akt signaling and leads to endothelial cell proliferative dysfunction. Amer J Physiol Heart Circ Physiol 2005; 289 (4): H1744-H1751.

7. Pedram A, Razandi M, Levin ER. Extracellular signal-regulated protein kinase/Jun kinase cross-talk underlies vascular endothelial cell growth factor-induced endothelial cell proliferation. J Biol Chem 1998; 273 (41): 26722-26728.

8. Narasimhan P, Liu J, Song YS, Massengale JL, Chan PH. VEGF Stimulates the ERK 1/2 signaling pathway and apoptosis in cerebral endothelial cells after ischemic conditions. Stroke 2009; 40 (4): 1467-1473.

9. Lee IA, Lee JH, Baek NI, Kim DH. Antihyperlipidemic effect of crocin isolated from the fructus of Gardenia jasminoides and its metabolite crocetin. Biol Pharm Bull 2005; 28 (11): 2106-2110. 
10. Wu Y, Pan R, Geng P. The effect of Crocin against hypoxia damage of myocardial cell and its mechanism. Chin J Appl Pysiol 2010; 26 (4): 453-457.

11. Bie X, Chen Y, Zheng X, Dai H. The role of crocetin in protection following cerebral contusion and in the enhancement of angiogenesis in rats. Fitoterapia 2011; 82 (7): 997-1002.

12. Lee S, Park Y, Zhang C. Exercise training prevents coronary endothelial dysfunction in type 2 diabetic mice. American journal of biomedical sciences 2011; 3 (4): 241.

13. Sakamoto K, Goodyear LJ. Invited review: intracellular signaling in contracting skeletal muscle. Journal of Applied Physiology 2002; 93 (1): 369-383.

14. Iemitsu M, Maeda S, Jesmin S, Otsuki T, Miyauchi T. Exercise training improves aging-induced downregulation of VEGF angiogenic signaling cascade in hearts. Amer J Physiol Heart Circ Physiol 2006; 291 (3): H1290-H1298.

15. Hu FB, Stampfer MJ, Solomon C, Liu S, Colditz GA, Speizer FE, Willett WC, Manson JE. Physical activity and risk for cardiovascular events in diabetic women. Ann Intern Med 2001; 134 (2): 96-105.

16. Ke Z, Yip SP, Li L, Zheng X-X, Tong K-Y. The effects of voluntary, involuntary, and forced exercises on brain-derived neurotrophic factor and motor function recovery: a rat brain ischemia model 2011.

17. Ellison GM, Waring CD, Vicinanza C, Torella D. Physiological cardiac remodelling in response to endurance exercise training: cellular and molecular mechanisms. Heart 2011: heartjnl-2011-300639.

18. Tsalouhidou S, Petridou A, Mougios V. Effect of chronic exercise on DNA fragmentation and on lipid profiles in rat skeletal muscle. Exp Physiol 2009; 94 (3): 362-370.

19. Zhang M, Lv X-Y, Li J, Xu Z-G, Chen L. The characterization of high-fat diet and multiple low-dose streptozotocin induced type 2 diabetes rat model. Exp Diabet Res 2009; 2008.

20. Algandaby MM, Alghamdi HA, Ashour OM, Abdel-Naim AB, Ghareib SA, Abdel-Sattar EA, Hajar AS. Mechanisms of the antihyperglycemic activity of Retama raetam in streptozotocin-induced diabetic rats. Food Chem Toxicol 2010; 48 (8): 2448-2453.

21. McDonald J, Pilgram T. Nuclear expression of p53, p21 and cyclin D1 is increased in bronchioloalveolar carcinoma. Histopathology 1999; 34 (5): 439-446.

22. Waltenberger J. VEGF resistance as a molecular basis to explain the angiogenesis paradox in diabetes mellitus. Biochem Soc Transact 2009; 37 (6): 1167.

23. Sasso FC, Torella D, Carbonara O, Ellison GM, Torella M, Scardone M, Marra C, Nasti R, Marfella R, Cozzolino D. Increased vascular endothelial growth factor expression but impaired vascular endothelial growth factor receptor signaling in the myocardium of type 2 diabetic patients with chronic coronary heart disease. J Amer Coll Cardiol 2005; 46 (5): 827-834.

24. Olsson AK, Dimberg A, Kreuger J, Claesson-Welsh L. VEGF receptor signalling? In control of vascular function. Nature Rev Mol Biol 2006; 7 (5): 359-371.

25. Hong CC, Peterson QP, Hong J-Y, Peterson RT. Artery/vein specification is governed by opposing phosphatidylinositol-3 kinase and MAP kinase/ERK signaling. Curr Biol 2006; 16 (13): 1366-1372.

26. Shiojima I, Walsh K. Role of Akt signaling in vascular homeostasis and angiogenesis. Circulat Res 2002; 90 (12): 1243-1250.

27. Naito Z, Takashi E, Xu G, Ishiwata T, Teduka K, Yokoyama M, Yamada N, Sugisaki Y, Asano G. Different influences of hyperglycemic duration on phosphorylated extracellular signal-regulated kinase $1 / 2$ in rat heart. Exp Mol Pathol 2003; 74 (1): 23-32.

28. Jiang BH, Zheng JZ, Aoki M, Vogt PK. Phosphatidylinositol 3-kinase signaling mediates angiogenesis and expression of vascular endothelial growth factor in endothelial cells. Proc Nat Acad Sci 2000; 97 (4): 1749-1753.

29. Pons S, Martin V, Portal L, Zini R, Morin D, Berdeaux A, Ghaleh B. Regular treadmill exercise restores cardioprotective signaling pathways in obese mice independently from improvement in associated co-morbidities. J Mol Cell Cardiol 2013; 54: 82-89.

30. Ghorbanzadeh V, Mohammadi M, Dariushnejad H, Abhari A, Chodari L, Mohaddes G. Cardioprotective Effect of Crocin Combined with Voluntary Exercise in Rat: Role of Mir-126 and Mir-210 in Heart Angiogenesis. Arquivos Brasil Cardiol 2017; 109 (1): 54-62.

31. Ghorbanzadeh V, Mohammadi M, Dariushnejad H, Chodari L, Mohaddes G. Effects of crocin and voluntary exercise, alone or combined, on heart VEGF-A and HOMA-IR of HFD/STZ induced type 2 diabetic rats. J Endocrin Invest 2016; 39 (10): 1179-1186.

32. Hariri AT, Moallem SA, Mahmoudi M, Memar B, Hosseinzadeh H. Sub-acute effects of diazinon on biochemical indices and specific biomarkers in rats: protective effects of crocin and safranal. Food Chem Toxicol 2010; 48 (10): 2803-2808.

33. Qi Y, Chen L, Zhang L, Liu WB, Chen XY, Yang XG. Crocin prevents retinal ischaemia/reperfusion injury-induced apoptosis in retinal ganglion cells through the PI3K/AKT signalling pathway. Exp Eye Res 2013; 107: 44-51.

34. Kang C, Lee H, Jung ES, Seyedian R, Jo M, Kim J, Kim JS, Kim E. Saffron (Crocus sativus L.) increases glucose uptake and insulin sensitivity in muscle cells via multipathway mechanisms. Food Chem 2012; 135 (4): $2350-2358$.

35. Guha A, Harmancey R, Taegtmeyer $\mathbf{H}$. Nonischemic heart failure in diabetes mellitus. Curr Opinion Cardiol 2008; 23 (3): 241.

36. Oku A, Nawano M, Ueta K, Fujita T, Umebayashi I, Arakawa K, Kano-Ishihara T, Saito A, Anai M, Funaki M. Inhibitory effect of hyperglycemia on insulin-induced Akt/protein kinase B activation in skeletal muscle. Amer J Physiol Endocrinol Metab 2001; 280 (5): E816-E824.

37. Dalle S, Longuet C, Costes S, Broca C, Faruque O, Fontés G, Bataille D. Glucagon promotes cAMP-response element-binding protein phosphorylation via activation of ERK1/2 in MIN6 cell line and isolated islets of Langerhans. J Biol Chem 2004; 279 (19): 20345-20355.

38. Cheng SM, Ho TJ, Yang AL, Chen IJ, Kao CL, Wu FN, Lin JA, Kuo CH, Ou HC, Huang CY. Exercise training enhances cardiac IGFI-R/PI3K/ Akt and Bcl-2 family associated pro-survival pathways in streptozotocininduced diabetic rats. Internat J Cardiol 2013; 167 (2): 478-485.

39. Ryder JW, Fahlman R, Wallberg-Henriksson H, Alessi DR, Krook A, Zierath JR. Effect of Contraction on Mitogen-activated Protein Kinase Signal Transduction in Skeletal Muscle involvement of the mitogen-and stress-activated protein kinase 1. J Biol Chem 2000; 275 (2): 1457-1462.

40. Gandhi GR, Jothi G, Antony PJ, Balakrishna K, Paulraj MG, Ignacimuthu S, Stalin A, Al-Dhabi NA. Gallic acid attenuates high-fat diet fed-streptozotocin-induced insulin resistance via partial agonism of PPAR $\gamma$ in experimental type 2 diabetic rats and enhances glucose uptake through translocation and activation of GLUT4 in PI3K/p-Akt signaling pathway. Eur J Pharm 2014; 745: 201-16. 